\title{
Evaluation of Biocompatibility of an Etch-and-Rinse Adhesive System Based in Tertiary Butanol Applied in Deep Cavity
}

\author{
Gilvanely Cardoso Alves ${ }^{1, *}$ and Ana Paula Veras Sobral ${ }^{2}$
}

\author{
${ }^{1}$ Department of Restorative Dentistry, Faculty of Dentistry, University of Pernambuco, Camaragibe, 1650, Pernambuco, \\ Brazil; ${ }^{2}$ Department of Oral Pathology, Faculty of Dentistry, University of Pernambuco, Camaragibe, 1650, Pernam- \\ buco, Brazil
}

\begin{abstract}
The aim of this study was to evaluate biocompatibility of an etch-and-rinse adhesive system based in tertiary butanol applied in deep cavity human teeth with approximately $1 \mathrm{~mm}$ of remaining dentin by observing histological changes of the pulp tissue of humans at intervals of 01, 07, 14 and 21 days. Twenty third molars with indication for extraction from patients of both sexes, presenting no systemic alterations were used. Class I cavity was made deeper and then, XP BOND adhesive system and resin Filtek Z250 were applied. The sample was divided into four groups according to the time intervals between the application of adhesive system and extraction. Morphologic criteria analysed considered the presence of hyperemia, type of inflammatory cell response, organization of odontoblast cells layer, organization of pulp tissue and the presence or absence of bacteria. Data were submitted to Fisher Exact Test $\mathrm{p}>0.05$. We observed mild inflammatory infiltrate, preserved pulp tissue morphology, disorganization of the odontoblast layer in most specimens, as well as absence of bacteria at the intervals of 01, 07, 14 and 21 days. In some cases there was pulp hyperemia. The etchand-rinse adhesive system based in tertiary butanol showed satisfactory behavior in the conditions studied.
\end{abstract}

Keywords: Bacteria, biocompatible materials, dental adhesives, dental pulp, dentin, hyperemia.

\section{INTRODUCTION}

Adhesive systems contain cytotoxic substances in their formulation such as resin monomers, solvents, initiators, inhibitors, and sometimes charged particles [1]. The amines used as co-initiators in adhesive systems also have cytotoxic and mutagenic effects because they have the ability to generate free radicals [2].

It is important to know the chemical components of the adhesive system, so one can understand its behavior as each component of the adhesive has a specific function [3]. Thus, the results related to cytotoxicity of these systems in the literature, can be explained by differences in composition.

The detailed study of biocompatibility of monomers in adhesive systems has made the cytotoxic effect of dimethacrylate possible to detect [4]. Moreover, these unpolymerized monomers diffuse through the dentinal tubules reaching the pulp tissue which may cause an inflammatory reaction $[5,6]$.

Conventional adhesives systems require acid etching the surface of enamel or dentin as a separate step. Persistent inflammation was observed in response to application of a conventional adhesive in deep dentin [7]. The composition of the adhesive system plays an important role in the cytotoxic effect it produces [8]. The choice of adhesive system for clinical use should take into consideration its biocompatibility [9].

*Address correspondence to this author at the Department of Restorative Dentistry, Faculty of Dentistry, University of Pernambuco, Camaragibe, 1650, Pernambuco, Brazil; Tel: +55 81 3184-7659; Fax: +55 81 3184-7686; E-mail: gilvanely@bol.com.br
XP BOND is a conventional adhesive system that differs from traditional dental adhesives. This etch-and-rinse adhesive system is based on tertiary butanol and composed of three linked hydrophilic monomer: dipentaerythritol pentaacrylate monophosphate (PENTA), 2-hydroxyethylmethacrylate (HEMA) and butane-1,2,3,4-tetracarboxylic acid di-ester-2-hydroxyethyl methacrylate (TCB). In addition to nanoparticles that increase the bond strength to enamel and dentin, the marginal integrity reinforce the new hybrid layer. Both PENTAs as the TCB are adhesion promoters, increasing the chemical interaction between the monomer and the dental substrate, thereby ensuring high adhesion strength to the remaining tooth structure. The HEMA is another hydrophilic monomer added in order to increase the resin content in the adhesive and reduce evaporation. It can also forget the presence of UDMA, which increases the strength of the hybrid layer. Its molecular chain provides improved compatibility with water and adhesives, which increases the stability making it behave similarly in both wet and dry dentines. Moreover, it has the boiling point higher than that causing slow evaporation of the acetone and increasing the working time. The tertiary butanol also possesses higher molecular weight alcohols, which increases the strength without impairing the evaporation rate, it has a smaller amount of hydrogen between molecules. Compared to ethanol, tertiary butanol has the following advantages: high stability and compatibility, not chemically reacting with the resins such as ethanol; enables increased resin content in the adhesive, improving the resistance of the formed hybrid layer and higher molecular weight than ethanol facilitating the application. Presents the same quality of adhesion to dentine regardless of the degree of moisture left in the cavity after acid condi- 
tioning. Such feature facilitates the restoration technique [10]. XP BOND is an adhesive system released by the Brazilian National Agency for Sanitary Vigilance, but to the best of the knowledge of the authors no studies that show its effects on cells and / or pulp tissue. In this sense, this study aims to evaluate the biocompatibility of an etch-and-rinse adhesive system based in tertiary butanol applied in deep cavity with approximately $1 \mathrm{~mm}$ of remaining dentin by means of histological alterations of the pulp tissue of humans at intervals of $01,07,14$ and 21 days.

\section{MATERIALS AND METHODS}

This study was approved by the Ethics Committee in Human Research of the University of Pernambuco, UPE, Brazil (\#188/09).

The subjects in this study were selected among patients of the dental clinic of the University. Of both sexes, they presented no systemic diseases and were aged between 20 and 40 years.

Exclusion criteria were as follows: teeth with carious lesions, restorations on any surface, negative response to test thermal pulp sensitivity to cold, abrasion, erosion, attrition, abfraction, periodontal disease, spontaneous pain; impossibility of absolute isolation of the operative field; individuals with systemic diseases and chronic smokers.

The sample was composed of 20 healthy erupted third molars with complete root formation and indication for extraction. Teeth were obtained from patients who gave their permission by signing the informed consent form.

Each tooth examined was subjected to periapical and interproximal radiographs. The periapical radiograph was used for diagnosis. The interproximal radiograph was used to observe the degree of involvement of the dentin tissue. The teeth included in the sample were submitted to pulp sensitivity test (cold) using Endo Frost refrigerant (Roeko Coltène/Whaledent, Langenau, Germany).

Patients had their teeth previously rinsed with $10 \mathrm{ml}$ of chlorhexidine gluconate $0.12 \%$ for 1 minute. Then, prophylaxis with pumice and water using a Robinson brush followed. Afterwards, anesthetic with vasoconstrictor (Mepivacaine 2\% with epinephrine 1:100.000, DFL, Rio de Janeiro, $\mathrm{RJ}$, Brazil) was administered.

The distance from tip of cusp to the ceiling of the pulp chamber was measured on the radiographs with the endodontic ruler. This measure, subtracted from $1 \mathrm{~mm}$ indicated the amount of tooth structure to be removed, which was passed on to the single use diamond tipped bur $\mathrm{n}^{\circ} 1015$ (KG Sorensen, Baureri, SP, Brazil) by making a groove in the shank itself [11]. A silicone stopper set the working length.

Light and intermittent movements on the occlusal surface with the spherical diamond tip in high rotation allowed an occlusal wear three times larger than the diameter of tip of the diamond bur and plenty of air and water cooling [12].

After removal of enamel, the rest of the preparation was conducted with a 245 bur (SS White, Lakewood, New Jersey, USA) whose working length had been set at the calibration mark. This bur was discarded after single use. In this study, each tooth after cavity preparation was evaluated. The control is a state of the pulp after cavity preparation was performed.

The absolute isolation of the operative field was carried out and antisepsis of the whole rubber sheet/clip was made with $2 \%$ chlorhexidine gluconate.

Initially, the margin of the enamel was etched with $37 \%$ phosphoric acid (Dentsply Caulk, Milford, DE, USA), for $15 \mathrm{~s}$, then the dentine was etched for more $15 \mathrm{~s}$ after which the acid was removed with water jet for $15 \mathrm{~s}$, air jet was applied for $5 \mathrm{~s}$ and a drop of XP BOND adhesive system (Dentsply Caulk, Milford, DE, USA - batch number 149023B) was placed in the tooth. Twenty seconds afterwards a brief blast of air was applied in order to evaporate the solvent. Light curing was performed with halogen light (Ultralux, Dabi Atlante, Ribeirão Preto, SP, Brazil) previously measured with the radiometer (RD7 Ecel, Ribeirão Preto, SP, Brazil) using power density of $500 \mathrm{~mW} / \mathrm{cm}^{2}$ for 20 seconds.

The teeth were then restored with resin Filtek Z250 (3M ESPE, St. Paul, MN, USA) inserted by using the incremental technique and light cured for 20s with the halogen light Ultralux using power density set of $500 \mathrm{~mW} / \mathrm{cm}^{2}$. After the finalized restoration occlusal contacts were checked.

The distribution of groups was made in Arabic numerals in ascending order according to the time intervals between application of adhesive system and surgery (Table 1).

Table 1. Distribution of groups according to the time intervals between application of XP BOND adhesive system and surgery.

\begin{tabular}{|c|c|c|}
\hline Groups & Time intervals (days) & $\mathbf{N}^{\mathbf{o}}$ of teeth \\
\hline \hline G1 & 01 & 05 \\
\hline G2 & 07 & 05 \\
\hline G3 & 14 & 05 \\
\hline G4 & 21 & 05 \\
\hline
\end{tabular}

The teeth were extracted at intervals of $01,07,14$ and 21 days after the restorative procedures. Immediately after extraction, 2/3 of the apical portion of roots were cut with a sterile diamond bur $\mathrm{n}^{\circ} 3145$ (KG Sorensen, Barueri, SP, Brazil).

The teeth were demineralized in 5\% nitric acid for 15 days. The specimens were embedded in paraffin. The following were obtained cuts of $5 \mathrm{~mm}$ obtained with a microtome were stretched on glass slides. Two slides were prepared from each specimen and stained by Hematoxylin-Eosin (H/E) and Brown-Hopps (BH) [13].

In order to detect bacteria, a slide with a histological section of human tooth with caries in dentin and pulp necrosis was obtained from the files of the Laboratory of Oral Pathology in FOP/UPE and used as a positive control.

The slides were viewed under conventional light microscope (Nikon E-100, Melville, New York, USA). Inflammatory cell response, the organization of the pulp tissue and 
presence/absence of bacteria, were analyzed according to the criteria De Souza Costa, Hebling, Randall [14] used.

The results were statistically evaluated using the Fisher exact test (SPSS - Windows 13.0) for categorical variables, which verified the existence of an association with $95 \%$ confidence.

\section{RESULTS}

The results indicated the pulpal reaction only performed cavity preparation as a baseline. Morphological analysis was described according to the groups (G1, G2, G3, G4) to which the teeth belonged. In G1 (01 day) most specimens showed preserved pulp tissue morphology (Fig. 1a). However, two specimens of G1 showed a mild inflammatory infiltrate (plasma cell) and disorganization of the odontoblast layer, whereas two other specimens of G1 showed pulp hyperemia (Fig. 1b). With respect to G2 (07 days) most specimens had their pulp morphology preserved. One specimen of G2 exhibited a mild inflammatory infiltrate (plasma cells and lymphocytes) (Fig. 1c). As to G3 (14 days), histological aspects were similar to those in G1 and G2. Only in one specimen of G3, a mild inflammatory infiltrate (plasma cell and lymphocyte), disorganization of the odontoblast layer (Fig. 1d) and pulp hyperemia were observed. Regarding G4 (21 days), histological aspects were similar to those in G1, G2 and G3. Two specimens of G4 exhibited mild inflammatory infiltrate and disorganized odontoblast layer and one specimen of G4 showed hyperemia of the pulp. Brown-Hopps stain, used for detecting bacteria, was negative for all specimens (Fig. 1e).

In relation to inflammatory cell response, the inflammatory infiltrate was absent in $70 \%$ of specimens; in $30 \%$ of specimens which had their pulp tissue morphology preserved, a mild inflammatory infiltrate was observed. As to the organization of the pulp tissue, most specimens (95\%) presented disorganization of the odontoblast layer and normal pulp tissue after application of the XP BOND adhesive system.

Statistical analysis of morphological criteria using the Fisher's exact test showed inflammatory cell response (ICR) and the Organization of the pulp tissue (OPT) at intervals of

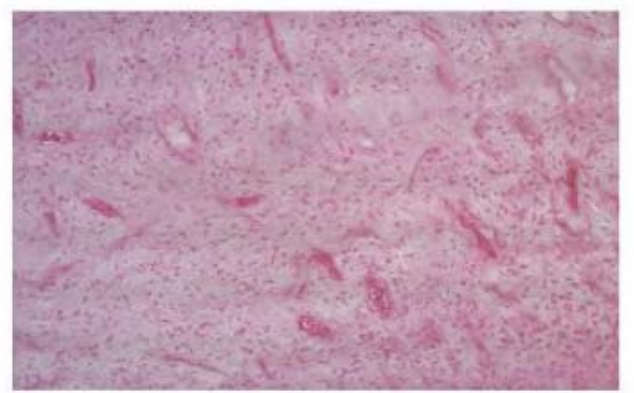

Fig. Ib.

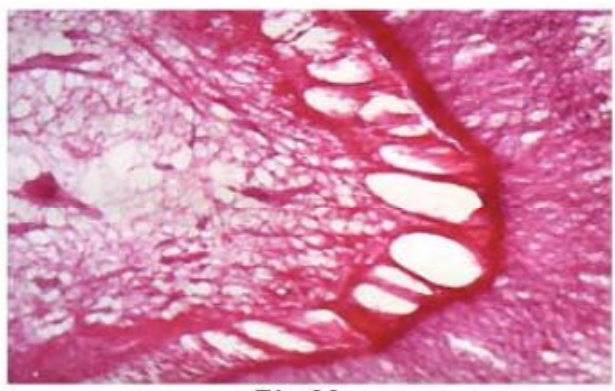

Fig. 1d.

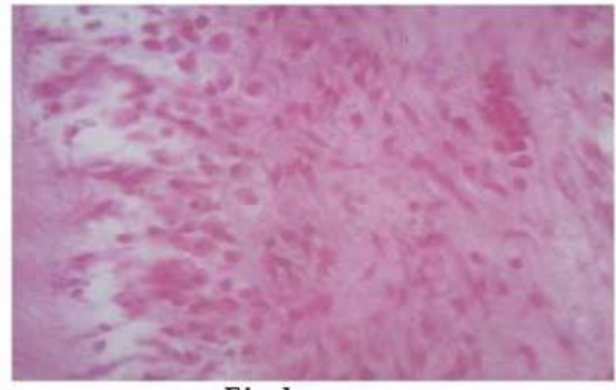

Fig. le.

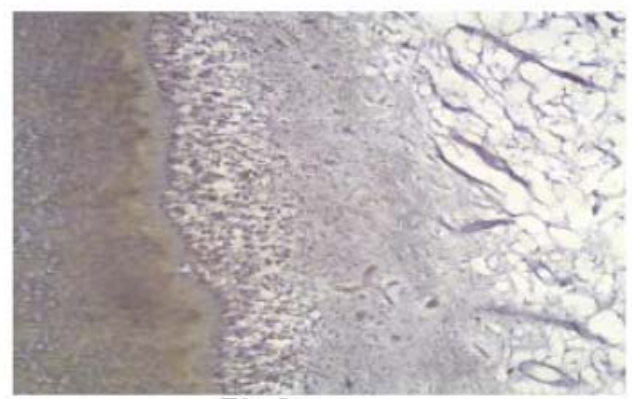

Fig. le.

Fig. (1). (a) Aspect of the preserved pulp tissue morphology, showing areas of degeneration of the odontoblast layer and mild inflammatory infiltrate (HE-40X). (b) Area of mononuclear cell infiltrate and congested vessels characterizing pulp hyperemia (HE-40X). (c) Inflammatory cells characterized by lymphocytes and plasma cells (HE-100x). (d) Hidrotic degeneration of the odontoblast layer (HE-100x). (e) Brown-Hopps negative (BH-40X). 
01, 07, 14 and 21 days (Table 2). Regarding ICR with inflammatory infiltrate absent, G1 (01 day) showed a rate of $60 \%$ with an increase to $80 \%$ in G2 (07 days) and G3 (14 days), returning to $60 \%$ in G4 (21 days). Regarding ICR, taking into account a mild inflammatory infiltrate, G1 (01 day) showed a percentage of $40 \%$ which decreased to $20 \%$ in G2 (07 days) and G3 (14 days) and increased to 40\% in G4 (21 days). Regarding OPT, there was disorganization of the odontoblast layer in $80 \%$ of specimens in G1 (01 day) and in $100 \%$ of them in the following groups: G2 (07 days), G3 (14 days) and G4 (21 days). The disorganization of pulp tissue occurred only in G1 (01 day) in $20 \%$ of specimens. Therefore, according to Fisher's exact test, no statistically significant association was found.

\section{DISCUSSION}

In biomaterials science, biocompatibility is a word very often used, but there is doubt about its real significance and the mechanisms of this phenomenon [15]. For example, the biocompatibility of the adhesive system, which promotes strong mechanical bond between the restorative material and tooth structure, still causes much discussion [16].

Both in vivo and in vitro studies have been made to assess the biocompatibility of dental adhesives. Most in vivo research has been performed on monkeys and rodents. In addition, there has also been in vitro cell culture tests [17]. The histological reaction of rodents is evaluated after using implants of adhesive systems in subcutaneous tissue [18]. In view of this interest in assessing the biological compatibility of adhesives, the need arose to study the biocompatibility of the XP BOND adhesive system, formerly approved by the Brazilian Agency for Sanitary Vigilance. The present study evaluated the XP BOND adhesive system in human pulp with remaining dentin approximately $1 \mathrm{~mm}$ away from pulp. Morphologic criteria analysed considered the presence of hyperemia, type of inflammatory cell response, organization of odontoblast cells layer, organization of pulp tissue and the presence or absence of bacteria.

In response to an infectious agent, the pulp triggers the inflammatory process, characterized in its early stage (stage vasoactive) by the excessive accumulation of blood in the vascular lumen, resulting in the congestion of vessels, known as pulp hyperemia. This condition was observed in two specimens of G1, one specimen of G3 and one specimen of G4 of this study. This finding characterizes a transient event and reversible pulpal pathology.

Any material applied on the teeth causes a response, which should be the least harmful possible for its cytotoxicity be considered low or nonexistent. This feature is essential for the classification of biomaterials. Because edema is a transient event, one can conclude that the pulp is physiologically able of responding to edema by returning to normal morphology. In this context, the adhesive tested - (XP BOND) - had a great performance. We consider that such response depends not only on the action of the material, but also on other conditions related to the host as well as to extrinsic factors, such as bacterial infection.

With respect to inflammatory cell response, XP BOND adhesive system triggered a mild inflammatory response having a small cell mobilization in defense of the pulp.

The materials used in dentistry may provoke defensive reactions (inflammatory reactions) in the flesh that vary with the type and intensity of the stimulus, ranging from hyperemia to pulp death. In response to mechanical stimuli such as dental cavities preparations and application of chemicals such as adhesives, the pulp may increase the blood flow and mobilize its defense cells, triggering an inflammatory process.

According to this study [19] the adhesive systems are biocompatible. However, another study [20] shows that the adhesives cause mild to severe inflammatory reactions in pulp tissue. This study [21] found that adhesive systems when applied to deep cavities in experimental conditions, proved to be biologically compatible.

Most of the specimens showed disorganization of the odontoblast layer after application of the adhesive system XP BOND. The odontoblasts are the cells most peripheral of the pulp, coating the entire length of the inner crown dentine and root. This particular type of cell is more susceptible to vari-

Table 2. Statistical analysis of morphological criteria.

\begin{tabular}{|c|c|c|c|c|c|}
\hline Variables & \multicolumn{4}{|c|}{ Time interval (days) } & p-value $(*)$ \\
\hline & n $(\%)$ & n $(\%)$ & n $(\%)$ & n $(\%)$ & \\
\hline \multicolumn{6}{|l|}{ ICR } \\
\hline Inflammatory infiltrate absent (Score 0 ) & $3(60,0 \%)$ & $4(80,0 \%)$ & $4(80,0 \%)$ & $3(60,0 \%)$ & 1,000 \\
\hline \multicolumn{6}{|l|}{ OPT } \\
\hline Disorganization of the odontoblast layer (Score 1) & $4(80,0 \%)$ & $5(100,0 \%)$ & $5(100,0 \%)$ & $5(100,0 \%)$ & 1,000 \\
\hline Disorganization of pulp tissue (Score 2) & $1(20,0 \%)$ & $0(0,0 \%)$ & $0(0,0 \%)$ & $0(0,0 \%)$ & \\
\hline
\end{tabular}

(*) Fisher's exact test

There was no statistically significant association in Table $\mathbf{2}$ 
ous external aggressions, including those arising from different clinical procedures or from the application of dental care materials, which can diffuse through the dentinal tubules reaching the pulp chamber.

This study [20] found no correlation between the presence of bacteria and the intensity of inflammatory reaction in their studies, but according to another study [22] there is a relationship between pulpal inflammation and the presence of bacteria and their products within the tubules. This study [23] and another study [24] corroborate what this study [22] stated. To these studies [2, 25] the components of adhesive systems are cytotoxic to pulp and pulpal injury occurs independently of the presence or absence of bacteria. These different results in the literature may be due to different compositions of materials and different application procedures.

The main constituents of the adhesive system are 2hydroxyethylmethacrylate (HEMA) and bisglicidil methacrylate (Bis-GMA). On a scale of cytotoxicity, HEMA, is less toxic in comparison with other monomers like BisGMA, urethane dime metacrylate (UDMA) and triethyleneglycol dimethacrylate (TEGDMA) after 24 and $72 \mathrm{~h}$ of exposure [26]. However, in high concentrations, HEMA can produce remarkable cytotoxic effects on cultured cells from the pulp [27]. Another equally important point is duration of exposure that has a strong effect on the toxicity of dental adhesives [28]. The XP BOND adhesive system has dimethacrylate HEMA, TEGDMA and UDMA in its composition, and, according to some studies all have cytotoxic effects [2, 10]. However, the adhesive system XP BOND triggered light inflammatory responses in the early stages and regression of inflammation in subsequent periods.

According to this study [28] the components in adhesive system alone or in combination may result in degenerative changes of the pulp when placed directly on exposed pulp. This is in line with study in which the capping was carried out with adhesive systems on human pulp [29]. The components of dentin bonding agents inhibit imunocompetent-cells proliferation, causing a chemical immunosuppression that favors the development of pulp pathologies [30].

According to our results and considering the thickness of the inflamed dentin we observed that the pulpal inflammatory response at the initial intervals either lacked inflammatory infiltrate or showed few inflammatory cells and no bacteria in most specimens what ultimately led to regression of the inflammatory process. This study [21] found that the intensity of the response of the dentin-pulp complex is related to the thickness of remaining dentin. We suggest that a remaining dentin thickness of approximately $1 \mathrm{~mm}$ appeared to be sufficient to avoid a noxious stimulus to the pulp when was applied the adhesive XP BOND.

Although results of mechanical testing have often been used as the criteria of choice for adhesives, biocompatibility should also be taken into consideration. Hence the importance of understanding the mechanisms related to biocompatibility. In this sense, future research on both the chemical and mechanical properties of adhesives are needed to assess their behavior. In addition, studies involving human subjects are vital for understanding the biological behavior and clinical success of the adhesive system.

\section{CONCLUSION}

According to the results of the present study, the following can be concluded: the conventional adhesive system based in tertiary butanol showed satisfactory behavior when applied approximately $1 \mathrm{~mm}$ away from the pulp chamber at intervals of $01,07,14$ and 21 days.

\section{CONFLICT OF INTEREST}

The authors confirm that this article content has no conflict of interest.

\section{ACKNOWLEDGEMENTS}

The authors thank the patients and Faculty of Dentistry, University of Pernambuco by facilities available for this study.

\section{REFERENCES}

[1] Nie J, Bowman CN. Synthesis and photopolymerization of N,N'dimethyl,-N,N'di(methacryloxy ethyl)-1,6-hexanediamine as a polymerizable amine coinitiator for dental restorations. Biomaterials 2002; 23: 1221-6.

[2] Kostoryz EL, Eick JD, Glaros AG, et al. Biocompatibility of hydroxylated metabolites of BISGMA and BFDGE. J Dent Res 2003; 82: 367-71.

[3] Van Landuyt KL, Snauwaert J, De Munck J, et al. Systematic review of the chemical composition of contemporary dental adhesives. Biomaterials 2007; 28: 3757-85.

[4] Volk J, Engelmann J, Leyhausen G, Geurtsen W. Effects of three resin monomers on the cellular glutathione concentration of cultured human gingival fibroblasts. Dent Mater 2006; 22: 499-505.

[5] Bianchi L, Ribeiro AP, Carrilho MR, Pashley DH, de Souza Costa CA, Hebling J. Cytotoxicity of adhesive systems of different hydrophilicities on cultured odontoblast-like cells. J Biomed Mater Res B Appl Biomater 2013; 101: 1498-1507.

[6] Lanza CR, de Souza Costa CA, Furlan M, Alécio A, Hebling J. Transdentinal diffusion and cytotoxicity of self-etching adhesive systems. Cell Biol Toxicol 2009; 25: 533-43.

[7] Tezvergil-Mutluay A, Mutluay M, Seseogullari-Dirihan R, et al. Effect of phosphoric acid on the degradation of human dentin matrix. J Dent Res 2013; 92: 87-91.

[8] Huang FM, Li YC, Lee SS, Chang YC. Cytotoxicity of dentine bonding agents on human pulp cells is related to intracellular glutathione levels. Int Endod J 2010; 43: 1091-7.

[9] da Silva JM, Rodrigues JR, Camargo $\mathrm{CH}$, et al. Effectiveness and biological compatibility of different generations of dentin adhesives. Clin Oral Investig 2014; 18: 607-13.

[10] Dentsply [homepage]. USA: XP BOND Directions for use; 2008 [cited 2008 Aug 22]. Available from: http:/www.dentsply.com.br/ hotsite/xpbond/bula.swf

[11] Leites AB, Baldissera EZ, Silva AF, et al. Histologic response and tenascin and fibronectin expression after pulp capping in pig primary teeth with mineral trioxide aggregate or calcium hydroxide. Oper Dent 2011; 36: 448-56.

[12] About I, Murray PE, Franquin JC, Remusat M, Smith AJ. The effect of cavity restoration variables on odontoblast cell numbers and dental repair. J Dent 2001; 29: 109-17.

[13] Brown RC, Hoops HC. Staining of bacteria in tissue sections. Reliable Gram stain method. Am J Clin Pathol 1973; 60: 234-40.

[14] de Souza Costa CA, Hebling J, Randall RC. Human pulp response to resin cements used to bond inlay restorations. Dent Mater 2006; 22: $954-62$.

[15] Williams DF. On the mechanisms of biocompatibility. Biomaterials 2008; 29: 2941-53.

[16] Ferracane JL, Cooper PR, Smith AJ. Can interaction of materials with the dentin pulp complex contribute to dentin regeneration? Odontology 2010; 98: 2-14.

[17] Kusdemir M, Gunal S, Ozer F, et al. Evaluation of cytotoxic effects of six self-etching adhesives with direct and indirect contact tests. Dent Mater J 2011; 30: 799-805. 
[18] Nagem-Filho H, Monteiro CR, Nagem HD, Lage-Marques JL. Effect of dental adhesives on the exudative phase of the inflammatory process in subcutaneous tissue of rats. Pesqui Odontol Bras 2003; 17: 109-12.

[19] Pereira SA, de Menezes FC, Rocha-Rodrigues DB, Alves JB. Pulp reactions in human teeth capped with self-etching or total-etching adhesive systems. Quintessence Int 2009; 40: 491-6.

[20] Gallorini M, Cataldi A, di Giacomo V. HEMA-induced cytotoxicity: oxidative stress, genotoxicity and apoptosis. Int Endod J 2014; 47: 813-8.

[21] Murray PE, Windsor LJ, Hafez AA, Stevenson RG, Cox CF. Comparison of pulp responses to resin composites. Oper Dent 2003; 28: 242-50.

[22] Tziafas D, Koliniotou-Koumpia E, Tziafa C, Papadimitriou S. Effects of a new antibacterial adhesive on the repair capacity of the pulp-dentine complex in infected teeth. Int Endod J 2007; 40: 5866.

[23] Büyükgüral B, Cehreli ZC. Effect of different adhesive protocols vs calcium hydroxide on primary tooth pulp with different remaining dentin thicknesses: 24-month results. Clin Oral Investig 2008; 12: 91-6.
[24] Cannon M, Cernigliaro J, Vieira A, Percinoto C, Jurado R. Effects of antibacterial agents on dental pulps of monkeys mechanically exposed and contaminated. J Clin Pediatr Dent 2008; 33: 21-8.

[25] Sengün A, Yalçın M, Ülker HE, Öztürk B, Hakkı SS. Cytotoxicity evaluation of dentin bonding agents by dentin barrier test on 3dimensional pulp cells. Oral Surg Oral Med Oral Pathol Oral Radiol Endod 2011; 112: e83-8.

[26] Grobler SR, Oliver A, Moodley D, Van Wyk Kotze TJ. Cytotoxicity of recent dentin bonding agents on mouse fibroblast cells. Quintessence Int 2008; 39: 511-6.

[27] Kurata S, Morishita K, Kawase T, Umemoto K. Cytotoxic effects of acrylic acid, methacrylic acid, their corresponding saturated carboxylic acids, HEMA, and hydroquinone on fibroblasts derived from human pulp. Dent Mater J 2012; 31: 219-25.

[28] Accorinte Mde L, Loguercio AD, Reis A, Muench A, de Araújo VC. Adverse effects of human pulps after direct pulp capping with the different components from a total-etch, three-step adhesive system. Dent Mater 2005; 21: 599-607.

[29] Sübay RK, Demirci M. Pulp tissue reactions to a dentin bonding agent as a direct capping agent. J Endod 2005; 31: 201-4.

[30] Huang FM, Li YC, Lee SS, Chang YC. Cytotoxicity of dentine bonding agents on human pulp cells is related to intracellular glutathione levels. Int Endod J 2010; 43: 1091-7.

(C) Alves and Sobral; Licensee Bentham Open.

This is an open access article licensed under the terms of the Creative Commons Attribution Non-Commercial License (http://creativecommons.org/licenses/by-nc/3.0/) which permits unrestricted, non-commercial use, distribution and reproduction in any medium, provided the work is properly cited. 